\title{
Is Pap Test Awareness Critical to Pap Test Uptake in Women Living in Rural Vietnam?
}

\author{
Hee Yun Lee ${ }^{1 *}$, Qingyi Li ${ }^{1}$, Yan Luo ${ }^{1}$, Kun Wang ${ }^{1}$, Sara Hendrix ${ }^{2}$, Jongwook \\ Lee $^{3}$, Sangchul Yoon ${ }^{4}$, Quoc Huy Nguyen $\mathrm{Vu}^{5}$
}

\begin{abstract}
Introduction: Cervical cancer is the second leading cause of cancer death among Vietnamese females. By detecting precancerous cells, Pap test screening plays a critical role in the fight against cervical cancer. The present study aims to investigate health-related factors associated with receipt of Pap test among Vietnamese females living in rural Vietnam, particularly examining the correlation between awareness level of the Pap test and the receiving of Pap test. Methods: Anderson's Behavioral Model of Health Services Use was utilized as the present study's theoretical framework. A selfadministrated questionnaire was completed among 193 females residing in Quantri City, Vietnam. Results: Only 15.5\% $(\mathrm{N}=30)$ of participants in our sample have had a Pap test. Pap test awareness $(\mathrm{OR}=18.38, \mathrm{p}<.001)$ was a strong predictor of Pap test receipt. Participants who had heard about Pap test were 18.38 times more likely to take a Pap test compared to those who had no prior knowledge. Besides the awareness, variables including employment $(\mathrm{OR}=.18, \mathrm{p}<.05)$, and health insurance coverage $(\mathrm{OR}=10.75, \mathrm{p}<.05)$ were significantly associated with Pap test uptake. Conclusion: Findings from the present study suggests interventions should be provided through public health efforts to enhance awareness of Pap test by aiming at increasing primary prevention of cervical cancer, especially among Vietnamese women living in rural areas, in order to reduce cancer health disparities.
\end{abstract}

Keywords: Pap test- Vietnamese women- Andersen Behavioral Model- cervical cancer screening- awareness

Asian Pac J Cancer Prev, 22 (3), 903-908

\section{Introduction}

Cervical cancer is the leading cancer and the second most common cause of death in Vietnamese females (Domingo et al., 2008). The incidence rate of cervical cancer in Vietnam affects 4.4 in every 100,000 women (Huynh et al., 2004). Although cervical cancer is a leading cause of death among women in developing countries, it is both preventable and curable (Suba et al., 2001). Globally, cervical cancer screenings have been shown to decrease cervical cancer incidence and mortality among women aged 21 to 65 (US Preventative Task Force, 2018).

Cervical cancer screening tests, such as the Papanicolaou test (Pap test), are often successful in identifying precancerous cells (American Cancer Society [ACS], 2018). Precancerous cells often go unnoticed due to being asymptomatic, which highlights the necessity of regular cervical cancer screenings (Smith et al., 2017). In Vietnam, preventative measures began in 1996 with a Memoranda of Understanding to begin Pap screening services for women, which sought to establish sustainable, cost-effective cervical cancer prevention services for women (Suba et al., 2001; Suba et al., 2006). To date, the cost of a Pap test and screening services remain inexpensive; however, the Pap test receipt in Vietnam still remains low (Domingo et al., 2008; Suba et al., 2001, 2006; Tran et al., 2018).

A number of sociodemographic factors are correlated with the uptake of Pap test screening including age, marital status, education, and income (Lee-Lin et al., 2007; Lee et al., 2015; Randolph et al., 2002; Akinlotan et al., 2018). Additionally, health insurance coverage has been associated with being the most important factor predicting Pap test screening (Do, 2015; Nguyen-Truong et al. ,2012; Luque et al., 2018). The knowledge of human papillomavirus (HPV), Pap test screening, and cervical cancers are also factors associate with Pap test screening ( Thompson et al., 2018; Ashtarian et al., 2017). Research has demonstrated a focus on cost-effectiveness analysis, quality management and sexual risk factors for uptake of the Pap test, and has limited findings in the role of health insurance or Pap test awareness (Suba et al., 2001; Suba

${ }^{1}$ School of Social Work, University of Alabama, Tuscaloosa, AL. ${ }^{2}$ Department of Health Sciences, University of Alabama, Tuscaloosa, AL. ${ }^{3}$ Department of Agricultural Economics and Rural Development, Seoul National University, Building, Seoul, Republic of Korea. ${ }^{4}$ Department of Global Health, Graduate School of Public School, Yonsei University, Seoul, South Korea. ${ }^{5}$ Hue University of Medicine \& Pharmacy, Hue, Vietnam.*For Correspondence: hlee94@ua.edu 
et al., 2005; Suba et al., 2006; Kim, Kobus et al., 2008; Tran et al., 2015).

In this study, Andersen's Behavioral Model of Health Service Utilization was used as framework to investigate factors related to Vietnamese's uptake of Pap test. Andersen's Behavioral Model of Health Service Utilization was a widely used model to explain factors associated with the utilization of health services (Andersen, 1995). Determinants of health service utilization could be categorized into three dimensions: predisposing factors, enabling factors and need factors (Andersen, 1995). In this study, predisposing factors are demographic information that cannot or can hardly be changed, such as age, marital status, employment status, and education level; enabling factors are those highly mutable factors, such as health insurance coverage, cervical cancer literacy, usual medical care place, and Pap test awareness; need factors include perceived and actual needs, such as family cancer history, personal cancer history, and self-rated health status.

To our knowledge, the present study is the first in exploring the role of Pap test awareness in uptake of Pap test screening among Vietnamese women. Therefore, it is imperative to investigate the relationship between healthrelated behavior factors and Pap test receipts in Vietnam. Considering the research gap on the factors of Pap test uptake and the association between Pap test awareness and Pap test receipt among Vietnamese women, the purpose of this study was to examine health-related factors associated with receipt of Pap test among Vietnamese women, particularly the correlation between awareness level of the Pap test and the receipt. The study addresses the following research questions:

- What is the level of Pap test receipt among the population of Vietnamese women?

- What are the risk factors associated with the receipt of Pap test?

- What is the relationship between Pap test awareness and the screening receipt after relevant variables are controlled?

\section{Materials and Methods}

\section{Research Design and Data Collection}

This study was part of a larger research program on the investigation of the prevention behaviors of different cancers, such as breast cancer, colorectal cancer, and cervical cancer in Vietnam. Collaborated with a local organization, a convenient sample of Vietnamese aged 18 or older were collected in rural areas in Quang Tri Province in 2017. A cross-sectional research design was adopted in this study. The self-administered questionnaire took 20-30 to complete. Before starting the questionnaire, informed consent was obtained from participants. A total of 193 Vietnamese women were recruited, and one participant aged over 65-year old was excluded from our study based on the population recommendation of U.S. Preventive Services Task Force. Thus, 192 participants were used for analysis. This study was approved by the Institutional Research Board (IRB) at the authors' university.

\section{Instrument}

Dependent variable. The outcome variable of this study was the receipt of Pap test, which was measured by a dichotomous value $(0=$ no, $1=$ yes $)$.

Independent variables. We utilized Andersen Behavioral Model to categorize independent variables which were divided into predisposing, enabling, and need variables.

Predisposing variables. Age, marital status, employment, and education level were selected as predisposing variables. Age was a continuous variable in our study. Dichotomous values were used for marital status $(0=$ others, $1=$ married or partnered $)$, employment $(0=$ no, $1=$ yes $)$, and education $(0=<$ Bachelor's degree, $1=\geq$ Bachelor's degree).

Enabling variables. Health insurance coverage, cervical cancer literacy, having usual medical care place, Pap test awareness were entered to enabling variable category. Yes/no questions $(0=$ no, $1=$ yes $)$ were conducted to measure whether having health insurance coverage, having usual medical care place, and having Pap test awareness. Cervical cancer literacy was measured by a 5 -item scale. Each of the items was a true/false question ( $0=$ false, $1=$ true), and the number of correct answers was the cervical cancer literacy score, which was treated as a continuous variable (0-5).

Need variables. Need variables included family cancer history, personal cancer history, and self-rate health status. Personal cancer history and family cancer history were dichotomized ( $0=$ no, $1=$ yes). A 5-point Likert scale was used to measure self-rated health status and the self-rated health score was regarded as a continuous variable (0-4) in our study.

\section{Data Analysis}

Univariate analysis was used to examine sociodemographic characteristics (age, marital status, employment, and education level), health accessibility (health insurance coverage, and having usual medical care place), health history information (family cancer history, personal cancer history, and self-rated health score) and health literacy information (Pap test awareness, and cervical cancer literacy). Bivariate analysis was conducted to test the relationship between these variables and the receipt of Pap test. Chi-square test was used for categorical variables, such as marital status, employment, education level, health insurance coverage, having usual medical care place, family cancer history, personal cancer history, and Pap test awareness. T-test was performed for continuous variables, such as age, self-rated health score and cervical cancer literacy. Logistic regression analysis predicting Pap test receipt was conducted with predisposing, enabling, and need variables. All analysis of this study was conducted using SPSS 25.0.

\section{Results}

\section{Participant Characteristics}

A total of 192 Vietnamese women were analyzed in our study. Nearly half (49.5\%) participants were aged 21-39 years, and the other half (50.5\%) were aged 40-59 
Table 1. Sociodemographic Characteristics of the Sample $(\mathrm{N}=192)$

\begin{tabular}{|c|c|c|c|c|c|}
\hline \multirow[t]{2}{*}{ Characteristic } & \multirow[t]{2}{*}{$\mathrm{n}$} & \multirow[t]{2}{*}{$\%$} & \multicolumn{3}{|c|}{ Received Pap Test } \\
\hline & & & $\mathrm{n}$ & $\%$ & \\
\hline \multicolumn{6}{|l|}{ Age } \\
\hline \multicolumn{6}{|l|}{$(\mathrm{M}=40.70, \mathrm{SD}=5.357)$} \\
\hline 21-39 years & 95 & 49.5 & 17 & 17.9 & 0.735 \\
\hline 40-59 years & 97 & 50.5 & 13 & 13.4 & \\
\hline \multicolumn{6}{|l|}{ Marital Status } \\
\hline Married or partnered & 176 & 91.7 & 27 & 15.3 & 0.129 \\
\hline Other & 16 & 8.3 & 3 & 18.8 & \\
\hline \multicolumn{6}{|l|}{ Employed } \\
\hline Yes & 171 & 89.1 & 26 & 15.2 & 0.21 \\
\hline No & 21 & 10.9 & 4 & 19 & \\
\hline \multicolumn{6}{|l|}{ Education Level } \\
\hline$<$ Bachelor's degree & 159 & 82.8 & 20 & 12.6 & $6.512 *$ \\
\hline Bachelor's degree & 33 & 17.2 & 10 & 30.3 & \\
\hline \multicolumn{6}{|c|}{ Health insurance coverage } \\
\hline Yes & 157 & 81.8 & 29 & 18.5 & $5.293^{*}$ \\
\hline No & 35 & 18.2 & 1 & 2.9 & \\
\hline \multicolumn{6}{|c|}{ Having usual medical care place } \\
\hline Yes & 125 & 65.1 & 21 & 16.8 & \\
\hline No & 67 & 34.9 & 9 & 13.4 & 0.375 \\
\hline \multicolumn{6}{|l|}{ Family cancer history } \\
\hline Yes & 49 & 25.5 & 9 & 18.4 & 0.375 \\
\hline No & 143 & 74.5 & 21 & 14.7 & \\
\hline \multicolumn{6}{|l|}{ Personal cancer history } \\
\hline Yes & 10 & 5.2 & 1 & 10 & 0.253 \\
\hline No & 182 & 94.8 & 29 & 15.9 & \\
\hline \multicolumn{6}{|l|}{ Pap test awareness } \\
\hline Yes & 70 & 36.5 & 25 & 35.7 & $33.724 * *$ \\
\hline \multirow[t]{3}{*}{ No } & 122 & 63.5 & 5 & 4.1 & \\
\hline & M & SD & \multicolumn{3}{|c|}{ Received Pap Test } \\
\hline & & & M & SD & $\mathrm{t}$ \\
\hline Age & 40.7 & 5.357 & 39.87 & 4.688 & 0.931 \\
\hline Self-rated health score & 2.06 & 0.598 & 2.27 & 0.64 & -1.978 \\
\hline Cervical cancer literacy & 2.73 & 1.601 & 2.77 & 1.073 & -0.162 \\
\hline
\end{tabular}

years. The majority of the women $(91.7 \%)$ were married or partnered and employed (89.1\%). Only $17.2 \%$ women held bachelors' degree or higher. With regard to health access, $81.8 \%$ of our participants were covered by health insurance, $65.1 \%$ had a usual medical care place. For health information, about $5.2 \%$ had personal cancer history, $25.5 \%$ had family cancer history and only $36.5 \%$ had ever heard about Pap test.

As Table 1 indicates, among those who received Pap test, significant differences were found in education $\left(\chi^{2}\right.$ $=6.512, \rho<.05)$, health insurance coverage $\left(\chi^{2}=5.293\right.$, $\rho<0.05)$, and Pap test awareness $\left(\chi^{2}=33.724, \rho<0.001\right)$. There were no significant differences in age, marital status, employment, having usual medical care place, personal cancer history and family cancer history. In addition, no significant differences were found in self-rated health score and cervical cancer literacy.

\section{Receipt of Pap Test by age and Time Frame}

As shown in Table 2, 15.5\% $(\mathrm{N}=30)$ participants in our samples had a Pap test in their life time, among which $17.9 \%(\mathrm{~N}=17)$ were aged $21-39$ years and $13.4 \%(\mathrm{~N}=13)$ were aged $40-59$ years. About $13 \%$ of participants aged from 21-39 years $(\mathrm{N}=12)$ and 3\% of participants in 40-59 years group $(\mathrm{N}=8)$ had Pap test within 3 years. Moreover, only $4 \%$ of all participants had Pap test within in a year in the present study. Approximately $5 \%$ of participants in 21-39 years $(\mathrm{N}=5)$ and $40-59$ yeas $(\mathrm{N}=5)$ took Pap test more than 3 years ago, respectively.

\section{Logistic Regression Analysis on Pap Test Receipt}

Among the predisposing variables, only employed $(\mathrm{OR}=.184, \mathrm{p}<0.05)$ were significantly associated with Pap test receipt. Participant who were employed showed significant lower likelihood of uptake Pap test in the present study. As for the enabling factors, health insurance coverage $(\mathrm{OR}=10.749, \mathrm{p}<0.05)$ was significant. Those who had health insurance coverage were 10.75 times that of those who do not have insurance to take Pap tests. Pap test awareness $(\mathrm{OR}=18.377, \mathrm{p}<0.001)$ was a strong significant predictor. Participants who were aware of Pap test were 18.38 times that of those who were not aware of it to take Pap test. All of the need variables were not significantly associated with receipt of Pap tests.

\section{Discussion}

The present study investigated the level of Pap test receipts and health-related factors associated with Pap test uptake among Vietnamese females guided by Andersen's Behavioral Model of Health Services. This study provided the first empirical evidence about the low level of Pap test receipt among females in Vietnam. Approximately $16 \%$ of participants have had a Pap test in their lifetime and only $4 \%$ of participants received a Pap test within a year, in the

Table 2. Receipt of Pap Test by Age and Time Frame (N=192)

\begin{tabular}{lccccccccc}
\hline & \multicolumn{3}{c}{ 21-39 $(\mathrm{n}=92)$} & \multicolumn{3}{c}{ Age } & \multicolumn{3}{c}{ Total } \\
Time & $\mathrm{n}$ & $\%$ & Cum. & $\mathrm{n}$ & $\%$ & Cum & $\mathrm{n}$ & $\%$ & Cum \\
\hline Ever had & 17 & 17.9 & & 13 & 13.4 & & 30 & 15.5 & 4.1 \\
1 year & 5 & 5.3 & 5.3 & 3 & 3.1 & 3.1 & 8 & 4.1 & 4.1 \\
1, 2 years & 5 & 5.3 & 10.6 & 3 & 3.1 & 6.2 & 8 & 4.1 & 8.2 \\
2, 3 years & 2 & 2.1 & 12.7 & 2 & 2.1 & 8.3 & 4 & 2.1 & 10.3 \\
3 years & 5 & 5.3 & 17.9 & 5 & 5.2 & 13.4 & 10 & 5.2 & 15.5 \\
\hline
\end{tabular}


Table 3. Logistic Regression Analysis on Pap Test Uptake ( $\mathrm{N}=192)$

\begin{tabular}{|c|c|c|c|c|}
\hline \multirow[t]{2}{*}{ Factors } & \multirow[t]{2}{*}{ Variables } & \multicolumn{3}{|c|}{ Received Pap test } \\
\hline & & $\mathrm{B}$ & OR & $95 \% \mathrm{CI}$ \\
\hline \multirow[t]{6}{*}{ Predisposing } & Age (29 54) & -0.085 & 0.919 & {$[0.831,1.015]$} \\
\hline & Marital status & -1.101 & 0.332 & {$[0.063,1.745]$} \\
\hline & $(\mathrm{Ref}=$ not married or partnered $)$ & & & \\
\hline & Employed $(\mathrm{Ref}=$ not employed $)$ & -1.691 & $.184 *$ & {$[0.034,0.994]$} \\
\hline & Education level & 0.425 & 1.529 & {$[0.483,4.840]$} \\
\hline & (Ref $=$ less than bachelor's degree) & & & \\
\hline \multirow[t]{4}{*}{ Enabling } & Health insurance coverage $(\operatorname{Ref}=$ no $)$ & 2.375 & $10.749 *$ & {$[1.189,97.187]$} \\
\hline & Cervical cancer literacy $(0 \sim 5)$ & -0.124 & 0.884 & {$[0.619,1.262]$} \\
\hline & Having usual medical care place $(\operatorname{Ref}=$ no) & -0.186 & 0.83 & {$[0.284,2.429]$} \\
\hline & Pap test awareness $(\operatorname{Ref}=$ no $)$ & 2.911 & $18.377^{* *}$ & {$[5.409,62.439]$} \\
\hline \multirow[t]{3}{*}{ Need } & Family cancer history $(\mathrm{Ref}=\mathrm{no})$ & -0.2 & 0.818 & {$[0.281,2.381]$} \\
\hline & Personal cancer history $(\mathrm{Ref}=\mathrm{no})$ & -0.94 & 0.391 & {$[0.036,4.211]$} \\
\hline & Self-rate health score $(0 \sim 4)$ & 0.4 & 1.491 & {$[0.684,3.251]$} \\
\hline \multicolumn{2}{|l|}{ Constant } & 0.011 & 1.011 & \\
\hline \multicolumn{2}{|l|}{ Pseudo $R^{2}$} & 0.394 & & \\
\hline \multicolumn{2}{|l|}{$\chi^{2}$} & \multicolumn{2}{|c|}{$9.347(p=0.314)$} & \\
\hline \multicolumn{2}{|c|}{-2 Log likelihood } & \multicolumn{2}{|l|}{116.678} & \\
\hline
\end{tabular}

Pseudo $R^{2}$ is Nagelkerke's $R^{2}$; B, coefficient; OR, odds ratio; ${ }^{*} p<0.05$, ${ }^{*} p<0.001$

present study. Vietnamese female participants who were employed showed a significantly lower level of Pap test receipt, which was consistent with the literature (Maxwell et al., 2000). This finding revealed that demographic characteristics are also important aspects to investigate and understand Vietnamese females who have not gotten the Pap test. One plausible explanation is that Vietnamese females who are employed tend to be occupied by their work and have less opportunities to receive cancer screenings than those who were unemployed. This result indicated that cancer prevention programs should target both employed and unemployed females to increase Pap test receipt.

Among enabling factors, participants who had health insurance revealed 10 times higher likelihood to receive a Pap test than those who were not insured among Vietnamese females in the present study, which has mirrored previous studies (Do, 2015; Yi et al., 2013). This result indicates essential implications in increasing the coverage of health insurance, thus improving cancer preventive behaviors and reducing health disparities in Vietnam.

More importantly, Vietnamese female participants who had heard of Pap tests or were aware of the Pap test was the strongest factor associated with the receipt of Pap tests which is in line with prior studies (Lam et al., 2003; Nguyen et al., 2012). Vietnamese females who have heard of Pap test were 18 times more likely to receive a Pap test compared to those who have never heard of one. This finding indicates that Pap test awareness is crucial for improving the Pap test receipt among females in Vietnam.

\section{Implications for Nursing Practice and Research}

Despite the limitations of the present study, a number of significant implications for nursing and health care practice and policy surfaced. The findings highlighted the importance of studying Vietnamese females, as well as studying their cultural and contextual factors related to health and cancer preventive behaviors. The present study identified two significant modifiable barriers to Pap test receipt in Vietnam, (1) the awareness of Pap test and (2) owning health insurance, which have fundamental implications for health practice and policy.

First, a lack of awareness or knowledge of the Pap test is the most critical barrier of getting a Pap test in Vietnam (Lam et al., 2003; Nguyen et al., 2012). Community-based educational programs could be essential to increase the Pap test receipt. Health message delivery through media campaigns and social network sites (e.g., Facebook or twitters) offer an innovative future intervention to increase the awareness of cervical cancer and the Pap test in Vietnamese communities. More importantly, future studies and practice should also pay attention on how to transfer the awareness of cervical cancer screening to an actual Pap test receipt in Vietnam (Fang et al. , 2011). Second, having health insurance could promote Pap test receipts among Vietnamese females. The present findings suggest that expanding health insurance coverage and increasing the financial accessibility of the Pap test is essential for Vietnamese females (Do, 2015). Finally, the findings presented in the current study provide the opportunity for nurses and health care professionals to explore and implement strategies to close the health disparity gap among adult women living in rural Vietnam.

\section{Limitations}

Although the present study provides valuable insight into health-related factors associated with receiving Pap tests among Vietnamese females, several limitations should be considered. First, the present study was a cross- 
sectional study which did not reveal causal relationships between factors and Pap test receipt. Next, the present study employed a small sample size of females in Vietnam. Therefore, the findings of this study need to be cautious when generalizing to Vietnamese females in Vietnam. However, the small sample size might not be a significant limitation due to the present results being consistent with previous studies. Future longitudinal studies would be needed to examine the causal relationships between health-related factors and the Pap test receipt among Vietnamese females and other Asian females.

In Conclusion, cervical cancer is the most common cancer among females in Vietnam which should be addressed by public health policies. Public health-related policies should advocate the screening for early detection, develop new techniques for cervical cancer diagnosis, and form a national medical network for cervical cancer prevention in Vietnam (Domingo et al., 2008). Literature supports that many women may be ignoring the importance of Pap testing, which additionally emphasizes the need for educational programs (Hsu et al., 2011). This study provides a step toward a better understanding of the barriers to Pap test receipts in Vietnam. The present study highlights the significant role of Pap test awareness. It is necessary and urgent for public health efforts to be focused on increasing the primary prevention of cervical cancer. This can be achieved by focusing on increasing the awareness of Pap tests in communities in order to reduce cervical cancer screening disparities in Vietnam (Domingo et al., 2008; Fang et al., 2011).

\section{Author Contribution Statement}

$$
\text { None. }
$$

\section{Acknowledgements}

This study has been approved by the University of Minnesota Institutional Review Board (IRB \#: 1604E87149) and the National Medical Center South Korea Institutional Review Board (IRB \#: H-1610071-001). This work has been supported by the Yonsei University College of Medicine grant fund ( 6-2014-0054) and Endowed Academic Chair Research Fund by the University of Alabama School of Social Work. This is not part of an approved thesis and no conflicts of interest.

\section{Conflicts of Interest}

The authors have no conflicts of interest relevant to this article to disclose.

\section{References}

American Cancer Society. (2018). Cervical Cancer. Retrieved from https://www.cancer.org/cancer/cervical-cancer/causesrisks-prevention/prevention.html.

Akinlotan MA, Weston C, Bolin JN (2018). Individual-and county-level predictors of cervical cancer screening: A multi-level analysis. Public Health, 160, 116-24.

Ashtarian H, Mirzabeigi E, Mahmoodi E, Khezeli M (2017). Knowledge about cervical cancer and Pap smear and the factors influencing the pap test screening among women. Int J Community Based Nurs Midwifery, 5, 188.

Do M (2015). Predictors of cervical cancer screening among Vietnamese American women. J Immigr Minor Health, 17, 756-64.

Domingo EJ, Noviani R, Noor MRM, et al (2008). Epidemiology and prevention of cervical cancer in Indonesia, Malaysia, the Philippines, Thailand and Vietnam. Vaccine, 26, M79.

Fang CY, Ma GX, Tan Y (2011). Overcoming barriers to cervical cancer screening among Asian American women. $N \mathrm{Am} J$ Med Sci, 4, 77.

Huynh MLD, Raab SS, Suba EJ (2004). Association between war and cervical cancer among Vietnamese women. Int $J$ Cancer, 110, 775-7.

Hsu Y, Cheng Y, Hsu K, Fetzer S, Chou C (2011). Knowledge and beliefs about cervical cancer and human papillomavirus among Taiwanese undergraduate women. Oncol Nurs Forum, 38, 297-304.

Kim JJ, Kobus KE, Diaz M, et al (2008). Exploring the costeffectiveness of HPV vaccination in Vietnam: Insights for evidence-based cervical cancer prevention policy. Vaccine, 26, 4015-24.

Lam TK, Mc Phee SJ, Mock J, et al (2003). Encouraging Vietnamese-American women to obtain pap tests through lay health worker outreach and media education. J Gen Intern Med, 18, 516-24.

Lee HY, Yang PN, Lee DK, Ghebre R (2015). Cervical cancer screening behavior among Hmong-American immigrant women. Am J Health Behav, 39, 301-7.

Lee-Lin F, Pett M, Menon U, et al (2007). Cervical cancer beliefs and Pap test screening practices among Chinese American immigrants. Paper presented at the Oncol. Nurs Forum, 34.

Luque JS, Tarasenko YN, Li H, et al (2018). Utilization of cervical cancer screening among Hispanic immigrant women in coastal South Carolina. J Racial Ethn, 5, 588-97.

Maxwell AE, Bastani R, Warda US (2000). Demographic predictors of cancer screening among Filipino and Korean immigrants in the United States. Am J Prev Med, 18, 62-8.

Nguyen-Truong CK, Lee-Lin F, Leo MC, et al (2012). A Community-Based participatory research approach to understanding pap testing adherence among Vietnamese American immigrants. J Obstet Gynecol, 41, E40.

Nguyen GT, Chen B, Chan M (2012). Pap testing, awareness, and acceptability of a human papillomavirus (HPV) vaccine among Chinese American women. J Immigr Minor Health, 14, 803-8.

Randolph WM, Freeman Jr DH, Freeman JL (2002). Pap smear use in a population of older Mexican-American women. Womens Health, 36, 21-31.

Smith RA, Andrews KS, Brooks D, et al (2017). Cancer screening in the United States, 2017: A review of current American cancer society guidelines and current issues in cancer screening. CA Cancer J Clin, 67, 100-121.

Suba EJ, Donnelly AD, Furia LM, Huynh MLD, Raab SS (2005). Coming to terms with Vietnam: The Viet/American cervical cancer prevention project. Diagn Cytopathol, 33, 344-51.

Suba EJ, Hung NC, Duc NB, Raab SS (2001). De novo establishment and cost-effectiveness of Papanicolaou cytology screening services in the socialist republic of Vietnam. Cancer, 91, 928-39.

Suba EJ, Murphy SK, Donnelly AD, et al (2006). Systems analysis of real-world obstacles to successful cervical cancer prevention in developing countries. Am J Public Health, 96, 480-7.

Thompson EL, Wheldon CW, Vamos CA, Griner SB, Daley EM (2018). How is health literacy related to Pap testing among US women?. J Cancer Educ, 34, 789-95. 
Hee Yun Lee et al

Tran BX, Than PTQ, Doan TTN, et al (2018). Knowledge, attitude, and practice on and willingness to pay for human papillomavirus vaccine: A cross-sectional study in Hanoi, Vietnam. Patient Prefer. Adherence, 12, 945.

Tran LT, Tran LT, Bui TC, et al (2015). Risk factors for highrisk and multi-type human papillomavirus infections among women in Ho Chi Minh City, Vietnam: A cross-sectional study. BMC Womens Health, 15, 16.

Yi JK, Lackey SC, Zahn MP, Castaneda J, Hwang JP (2013). Human papillomavirus knowledge and awareness among Vietnamese mothers. J Community Health, 38, 1003-9.

US Preventive Services Task Force. (2018). Screening for cervical cancer us preventive services task force recommendation statement. JAMA, 320, 674-86.

\section{(ब) $(\otimes$}

This work is licensed under a Creative Commons AttributionNon Commercial 4.0 International License. 